\title{
Quality-by-Design Development of a Patient Mobility e-Monitoring System
}

\author{
Yaël Kolasa ${ }^{1,2}$, Eliott Gandiole ${ }^{1,4}$, and Thierry Bastogne $e^{1,2,3}$ \\ 1 Université de Lorraine, CNRS, CRAN, F-54000 Nancy, France \\ 2 CYBERnano, F-54600 Villers-lès-Nancy, France \\ https://www. cybernano.eu/ \\ 3 INRIA SIMBA, F-54000 Nancy, France \\ 4 Université de Lorraine, F-54000 Nancy, France
}

\begin{abstract}
Wearable sensors are a growing trend within the Internet-ofThings. Their use within many fields, such as e-Health, is more and more common and with such versatility and constraints due to their portable nature, it is important to achieve the best fine-tuning possible for the desired application. To this end, the application of the Quality-by-Design good practices of development could drastically ease the process. This study aims to apply the Quality-by-Design approach to the development of an e-monitoring solution of patient mobility. After defining the profiles of the targeted system, three critical quality attributes: autonomy, data upload duration and data integrity are defined. A criticality assessment study, based on the implementation of specific designs of experiments, is carried out to identify the most impacting process parameters. Finally, suitable operating modalities of the sampling rate, number of sensors, as well as uploading mode and period are determined to comply with the specifications on the three critical quality attributes.
\end{abstract}

Keywords: Quality-by-Design · Internet of Things · Wearable sensors . e-Health.

\section{Introduction}

The ever growing use of Internet of Things nowadays has shed light on its potential for healthcare applications. Such versatility also comes with significant downsides that need to be monitored in order to produce an adequate solution. To help define and fine tune a solution to remain within specifications, the Quality by Design development guidelines (ICH Q8-Q12) ${ }^{5}$, can be used. Quality by Design $(\mathrm{QbD})$ is a data-driven engineering approach made to assess and control risks of development (being out-of-specifications) at the earliest steps by using appropriate statistical modeling methods. Since mid-2000, QbD has become the main good practices guidelines recommended by ICH (International Council for Harmonisation of technical requirements for pharmaceuticals for human use) for

\footnotetext{
${ }^{5}$ https://www.ich.org/
} 
drug development [12]. Similar risk-based development approaches was previously applied to control quality and safety during the whole product lifecycle such as the Total Quality Management in the United States Navy [3] and Six Sigma used by Motorola [4 in mid-1980, but also Lean Management [5] in mid1990. QbD has introduced the concept of "Design Space": a probabilistic map of quality and safety risks in the space of process parameters, which allows developers (i) to identify which are the most critical parameters to be controlled and (ii) to determine which are the normal operating regions in which critical parameters have to belong.

In this study, we assess the applicability of the first steps of the QbD workflow to the safe development of a patient mobility e-monitoring solution based on wearable inertial motion units. Indeed the $\mathrm{QbD}$ concept was initially proposed for drug development and our objective is to examine its applicability to implement a rational design of medical devices and more particularly e-Health systems. We firstly present the Quality-by-Design cycle before applying the first steps to our study case, and finally presenting and discussing the application results.

\section{Method}

\subsection{Quality-by-Design Approach}

Quality by Design (QbD) is a risk-based engineering approach that aims at reducing cost and time of development by minimizing additional unexpected resources not already allocated. The $\mathrm{QbD}$ workflow can be sum up by a cycle decomposed into eight key steps but only the first five ones were implemented in this study case.

1. The first step of QbD is to define the Quality Target Product Profile (QTPP): a dynamic summary of the expected quality features regarding the future product [6]. This proactive document can include the technical and economical characteristics of the future product. This document has to be updated throughout the development process. It is intended for all the stakeholders: management, board members, advisors, investors, regulatory authorities and strategic partners.

2. The second step aims at defining the Critical Quality Attributes (CQA) of the product. A CQA is a physical, chemical, biological or microbiological property or characteristics that should be within an appropriate range to ensure the desired product "quality" 6]. Multiple methods can be used to assess criticality of quality attributes. For instance, Summary Risk Chart can allow the identification of CQA with a simple traffic light color indicating the rank of the criticality (red for critical, amber for potentially critical and green for non-critical). The FMEA method (Failure Mode \& Effects Analysis) is another possible method to evaluate criticality of quality attribute.

3. In the third step, critical Material Attributes and Process Parameters are identified. They correspond to design and manufacturing factors, which have 
a significant effect on the critical quality attributes. They should be monitored or controlled to ensure that CQA specifications are fulfilled. A common tool used to enumerate all risk factors is the Ishikawa diagram.

4. A criticality assessment of process parameters is performed in a fourth step to identify the main active system parameters to focus on. Screening design of experiments such a Taguchi or Plackett-Burman designs are often implemented to that aim [6]. They allow to reduce a lot the number of experiments to be carried out and to rank their effects in a Pareto diagram as illustrated in Figure 1 .

5. The mathematical relationship between critical quality attributes (outputs) and critical process parameters (inputs) is identified in a fifth step in which specific experimental designs such as central composite, Box-Behnken and Doehlert are implemented to collect informative data with a parsimonious number of trials. In practice, response surface models are used to describe those relationships. This type of model allows us to perform prediction, optimization and to compute a Design Space, i.e. a diagram composed of four regions of probability:

- the Out Of Specification (OOS) region - outlines the region where the quality product is not acceptable and thus necessitates investigations in order to highlight the reasons of unacceptance;

- the Proven Acceptance Region (PAR) - depicts the region where an acceptable product can be produced but where some adjustments should be done to access the NOR;

- the Normal Operating Region (NOR) - represents the desired region where the quality product has a high degree of probability to comply with the quality specifications;

- the Control Operating Region (COR) - a subregion of NOR in which a control strategy has to maintain the operating point.

A design space can be regarded as a map locating the operating region of quality.

6. The sixth step introduces process analytical technology (PAT) to measure on-line critical attributes and process parameters. PAT often relies on process analyzers and sensors to inform in real-time about the location of the operating point within the Design Space.

7. The feedback information provided by PAT is necessary to implement a control strategy able to maintain the operating point within the COR region of the design space whatever the fluctuation of background variables. Statistical Process Control and Bayesian Process Control are two examples of strategy often used in practice to solve that technical issue [6].

8. The last step of QbD concerns the Data Management. All the manufacturing data can be stored in a large database, which can be used a posteriori to refine the working hypothesis and help to continuously improve the product quality.

In this application, only the first five steps are implemented since our objective is firstly to establish the proof of concept for the e-monitoring system. 


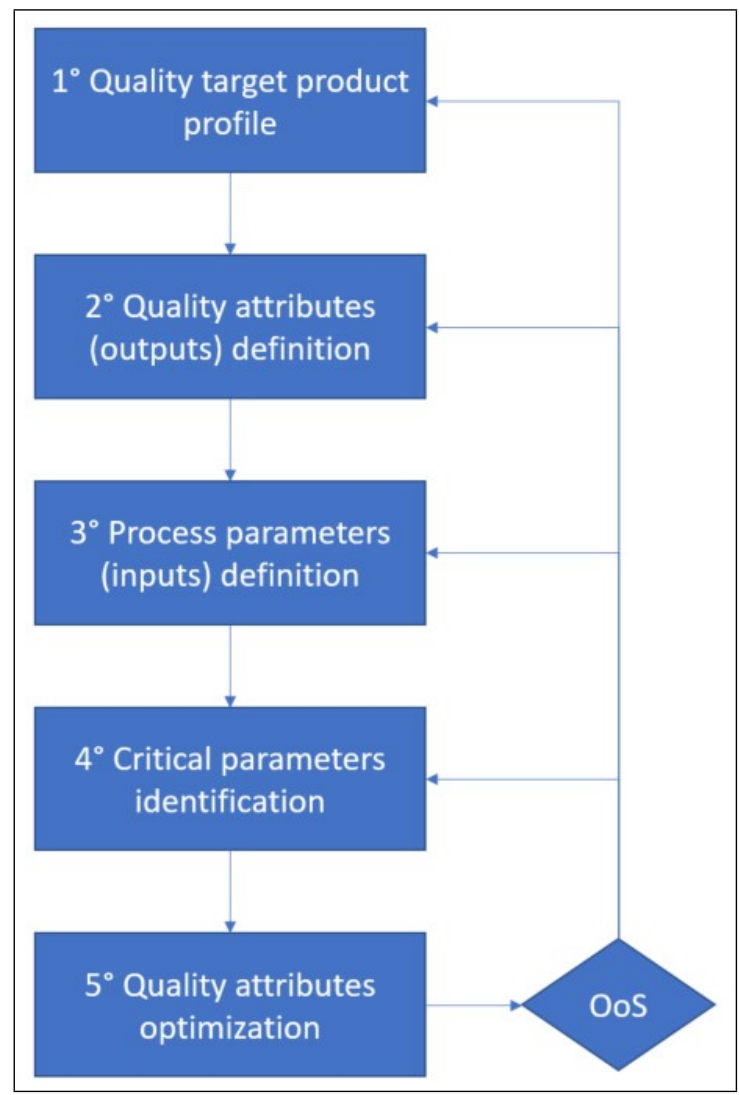

Fig. 1. Quality-by-Design methodology (OoS: Out of Specification)

Moreover, since most of the system parameters are categorial, the determination of a Design Space is not necessary since we can directly identify their optimal modalities.

\subsection{Quality Target Product Profile}

Intended application. The system to be developed aims at collecting, monitoring and characterizing the mobility of patients for targeted applications such as electronic Clinical Outcome Assessment (eCOA), Adapted Physical Activity (APA) or homecare monitoring. Targeted patients can be involved in clinical studies, or people with chronic conditions as well as elderly persons. The wearable sensor can be worn on the waist (belt), the wrist (bracelet), the foot (shoes) or on the ankle. The environment in which the device shall be used is the patient's home but could also be used in an hospital, a clinic or a retirement residence. 
Materials. The test subject was equipped with one wearable inertial sensor at his belt. We used a MetaMotionR sensor developed by Mbientlab Inc, as seen in Fig. 2. This technology is composed of a BMI160 6-axis Accelerometer + Gyroscope, a BMM150 3-axis Magnetometer, a BOSCH 9-axis Sensor Fusion, a 8MB Flash Memory and a lithium-ion rechargeable battery. 9 signals (accelerometry, gyrometry and magnetometry) were measured during each run. The Metabase mobile application proposed by Mbientlab was used to collect the experimental data. The design of experiments and the data analysis were performed in the $\mathrm{R}$ statistical computing environment.

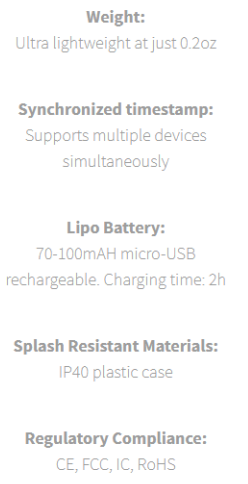

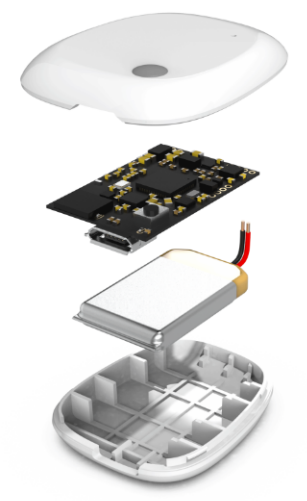

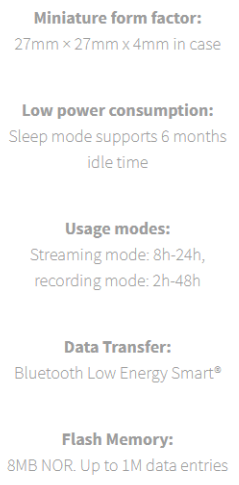

Fig. 2. MetaMotionR sensor (Mbientlab)

\subsection{Critical Quality Attributes}

To make such a patient mobility e-monitoring possible, some technical properties are critical such as the memory of the wearable sensor, and its autonomy. Another important quality criterion deals with the data integrity. Indeed, for a good monitoring of the patient mobility, it is critical to minimize data loss. Since no measurement is possible during the data uploading process, the duration of data transfer is also identified as a critical quality attribute. As a consequence, six critical quality attributes noted $Y_{1}$ to $Y_{6}$, have been defined in the table 1 with their desired specifications. The memory should hold up to at least 30 minutes of data, the battery must power up the sensor for at least 16 hours, the data uploading duration shall not exceed $30 \%$ of the data uploading period and the data integrity shall be greater than $80 \%$.

\subsection{Identification of Process Parameters}

The definition of Process Parameters is done in the third items of Fig. 1 A cause-effect (Ishikawa) diagram is used for each CQA to list all the potential 
Table 1. Critical quality attributes

\begin{tabular}{|c|c|c|c|}
\hline Variable & Factor & Unit & Specification \\
\hline \hline$Y_{1}$ & Memory & Time & $>30 \mathrm{~min}$ \\
\hline$Y_{2}$ & Autonomy & Time & $>16 \mathrm{~h}$ \\
\hline$Y_{3}$ & Data uploading Duration / Data uploading Period & $\%$ & $<30$ \\
\hline$Y_{4}$ & Data Integrity (Accelerometry) & $\%$ & $>80$ \\
\hline$Y_{5}$ & Data Integrity (Gyrometry) & $\%$ & $>80$ \\
\hline$Y_{6}$ & Data Integrity (Magnetometry) & $\%$ & $>80$ \\
\hline
\end{tabular}

causes that could affect the quality attributes of the medical device [7]. Causes are usually grouped into major categories to identify and classify these sources of variation.

Among all the listed factors, some of them cannot be controlled and are therefore considered as disturbances. Their effect will be described by a random variable in the risk analysis model $(E(k))$ in equation 1 . The remaining factors are split up into two groups: the factors under consideration will be gathered to be studied in the next step while the estimated irrelevant parameters will be kept constant during the experiments.

\subsection{Criticality Assessment of Process Parameters}

The parameters under consideration identified at the previous step are all involved in a criticality assessement process involving both a full factorial design of experiments and t-tests [6] . This analysis relies on an ANOVA model described as follows:

$$
Y_{i}(k)=b_{0}+b_{1 B} u_{1 B}(k)+\cdots+b_{p B} u_{p B}(k)+E(k),
$$

with:

$-Y_{i}(k)$ : measured value of the $i-t h$ CQA during trial $k$

- each factor $u$ takes two modalities: $A$ and $B$.

$-b_{0}$ : mean response when all factors takes their reference level (A).

$-b_{1 B}$ : additive effect of factor 1 when it takes its level B.

$-b_{p B}$ : additive effect of factor $p$ when it takes its level B.

$-E(k) \sim \mathcal{E}\left(0, \sigma^{2}\right)$ : random variable describing the experimental modeling residuals.

- Variance $\sigma^{2}$ represents the inter-assay variability.

An ANOVA estimator was used to determine the model coefficients: $b$ (factor effects) from the collected data on the response: $Y_{i}$. After the estimation step, a t-test is applied to each model coefficient to assess its statistical relevance, i.e. the criticality of the associated factor effect:

$$
\begin{cases}H_{0}: & b_{i}=0 \\ H_{1}: & b_{i} \neq 0\end{cases}
$$

At total seven factors were tested in this study: 
Table 2. Factors under consideration in the different tests carried out in this study. AGM: Accelerometer/Gyrometer/Magnetometer. STR: streaming and STO: data storage on sensor.

\begin{tabular}{|c|c|c||c|c|c|}
\hline Notation & Factor & Unit & Level 1 & Level 2 & Level 3 \\
\hline \hline$u_{1}$ & Sampling rate & Hz & Low Frequency & High Frequency & \\
\hline$u_{2}$ & Nb of Sensors & - & AG & AGM & \\
\hline$u_{3}$ & Data upload mode & - & STR & STO & \\
\hline$u_{4}$ & Data uploading period & min & 15 & 60 & \\
\hline$u_{5}$ & Bluetooth mode & - & ON & OFF during 1h & \\
\hline$u_{6}$ & Sensor/mobile phone distance & $\mathrm{m}$ & $<5 \mathrm{~m}$ & $>10 \mathrm{~m}$ & \\
\hline$u_{7}$ & Wall type & - & none & thin & thick \\
\hline
\end{tabular}

Tests on Autonomy and Memory Operating Duration The risks of having the autonomy of the sensor and the memory duration not meeting the expectation would be the loss of data collected by the device and therefore a lesser quality of diagnostic afterwards. Factors under consideration for the criticality assessement associated with the memory operating time are $u_{1}, u_{2}$ and $u_{3}$. The implemented experimental protocol consists in testing the most extreme combination of factors, i.e. $u_{1}$ : high frequency, $u_{2}$ : three sensors AGM and $u_{3}$ in storage mode.

Tests on upload time Factors under consideration for the criticality assessement associated with the upload time are presented in Table 2, The $2^{3}$ full factorial design of Memory tests is composed of eight different experimental conditions, each one duplicated once, and is presented in Table 3. We can see in the table 3 the three critical parameters that are to be tested, with the first being the sampling frequency of the sensors: $u_{1}$. This parameter is thought to heavily impact the autonomy and the memory availability of the device. The second is the number of sensors: $u_{2}$. We can see in the table 2 the characteristics of the different sensors available on the chip. Each one of them have a minimum and a maximum frequency which will also determine the electric consumption of the sensor, and the volume of data generated. Furthermore, the accelerometer and gyrometer $(\mathrm{AG})$ are either both set to work, or the additional magnetometer (AGM) can be added. The accelerometer and the gyrometer are both needed to have coherent data, the magnetometer adds useful but optional data. A third sensor will also add to the electric consumption and data generation of the system. The last parameter is the data uploading period: either 1 or 4 upload sessions every hour.

Tests on data integrity Factors under consideration for the criticality assessement associated with data integrity are: $u_{5}, u_{6}$ and $u_{7}$ defined in Table 2 . They are involved in a $2^{3}$ full factorial design composed of eight different experimental conditions and is presented in Table 4 . 


\section{Results}

\subsection{Criticality assessment of the Sensor Autonomy (Battery Duration)}

The Ishikawa diagram of the battery duration is presented in Figure 3(a). It shows the potential factors that may affect the sensor autonomy. Among them, three parameters, given in table 2 were selected. One experiment was carried out in extreme condition, i.e. by using three sensors (Accelerometer + Gyrometer + Magnetometer), each at high sampling frequency $(25 \mathrm{~Hz}, 50 \mathrm{~Hz}, 25 \mathrm{~Hz})$ and with a local data recording on the inertial motion unit. After $22 \mathrm{~h}$ of operation, the battery charge level was still at $53 \%$. In other terms, even in this critical configuration, the battery duration is enough to comply with the desired application.

Table 3. Full Factorial Design applied to the Data Upload Duration

\begin{tabular}{|c|c|c||c|}
\hline$u_{1}$ & $u_{2}$ & $u_{4}$ & $Y_{3}(\%)$ \\
\hline $\mathrm{BF}$ & $\mathrm{AGM}$ & 15 & 41.67 \\
$\mathrm{BF}$ & $\mathrm{AG}$ & 60 & 25.56 \\
$\mathrm{HF}$ & $\mathrm{AGM}$ & 15 & 39.78 \\
$\mathrm{HF}$ & $\mathrm{AG}$ & 15 & 36.00 \\
$\mathrm{HF}$ & $\mathrm{AGM}$ & 60 & 26.00 \\
$\mathrm{HF}$ & $\mathrm{AG}$ & 60 & 25.28 \\
$\mathrm{BF}$ & $\mathrm{AG}$ & 60 & 23.25 \\
$\mathrm{BF}$ & $\mathrm{AGM}$ & 60 & 24.58 \\
$\mathrm{BF}$ & $\mathrm{AG}$ & 15 & 32.22 \\
$\mathrm{HF}$ & $\mathrm{AG}$ & 60 & 26.67 \\
$\mathrm{BF}$ & $\mathrm{AGM}$ & 60 & 26.61 \\
$\mathrm{BF}$ & $\mathrm{AG}$ & 15 & 37.33 \\
$\mathrm{BF}$ & $\mathrm{AGM}$ & 15 & 53.78 \\
$\mathrm{HF}$ & $\mathrm{AG}$ & 15 & 48.11 \\
$\mathrm{HF}$ & $\mathrm{AGM}$ & 15 & 36.44 \\
$\mathrm{HF}$ & $\mathrm{AGM}$ & 60 & 23.17 \\
\hline
\end{tabular}

\subsection{Criticality assessment of the Memory Operating Duration}

If storage capacity cannot withhold all data generated within a session, the oldest data would be lost to the newest ones, thus lowering the quality of the data analysis once they are transferred. New data could also partially replace some old data, thus giving unreadable data that are still stored and taking place on the storage. The Ishikawa diagram of the Memory Operating duration is presented in Figure $3(\mathrm{~b})$. It shows the potential factors that may affect this quality operating attribute. As previously, the three parameters; $u_{1}, u_{2}$ and $u_{3}$ were tested and the same extreme experimental condition than the one implemented before to test the battery duration were used to address the question of the memory 
operating duration. In this case, the sensor memory was saturated after $1 \mathrm{~h} 26$. As a consequence, we have decided to fix the duration upload period to $30 \mathrm{~min}$ (i.e. 2 data upload session /h).

\subsection{Risks on Data Upload Duration}

The risks encountered on data upload duration is to miss an important motion event during the period of time. Indeed, the longer it takes to upload the data, the fewer data can be collected and analyzed because the device is busy sending them instead of collecting them. Thus, lowering the pertinence and the efficiency of the analysis algorithm. This can, at the end, provide faulty results. The Ishikawa diagram of the Memory Operating duration is presented in Figure 3(c)] Three specific factors were more particularly tested: $u_{1}, u_{2}$ and $u_{4}$. To that aim, the full factorial design presented in Table 3, were implemented. Estimation results are presented in the Pareto diagram of the Figure 4. It clearly shows that only $u_{4}$ (Data Uploading Period) has a significant effect. Increasing $u_{4}$ from $15 \mathrm{~min}$ to $60 \mathrm{~min}$ lowers $Y_{3}$. As a consequence, this factor will be fixed at $60 \mathrm{~min}$ in the sequel.

\subsection{Risks on Data Integrity}

If data integrity is not ensured, because of a lack of battery life or storage capacity, the analysis cannot take place, or could produce erroneous results. Nevertheless, four experimental conditions were not able to be carried out in the environment we had to conduct the trials. The observation dataset is given in Table 4. Responses can be greater than 100\%, in that case, it means that the sensor saved several times some pieces of data after transmissions interruptions. $100 \%$ corresponds to a perfect continuous communication without loss. Three specific factors were tested to estimate their effect on Data Integrity: $u_{5}, u_{6}$ and $u_{7}$. To that aim, the fractional factorial design presented in Table 4 , were implemented.

\subsection{Integrity of Accelerometer Data}

The estimated effects of each factor are presented in Figure 5(a) Only the first two effects were significant ( $t$-test: $p=0.0077$ and $p=0.042$ respectively). We can see that the most impacting factor is $u_{6}$, which implies that the further the smartphone is from the sensor, the lesser the communication is. We can also see that when the factor $u_{5}$ is OFF, i.e. when the Bluetooth mode is inactive, the response is better. $u_{7}$ has no significant effect on the integrity of accelerometric data.

\subsection{Integrity of Gyrometer Data}

The estimated effects of each factor are presented in Figure 5(b). The first two effects were almost significant (t-test: $p=0.071$ and $p=0.078$ respectively). We 
Table 4. Full Factorial Design and experimental data for the Data Integrity Test. $u_{5}$ : bluetooth mode (ON: normal / OFF: no bluebooth at the first connection but switched on and re-connection after $1 \mathrm{~h}) . u_{6}$ : sensor/mobile phone distance $(<5 \mathrm{~m} ;>10 \mathrm{~m}) . u_{7}$ : wall type (no wall, "Cloison"=thin wall, "Porteur"=thick concrete wall) $Y_{4}$ : \% data for accelerometer. $Y_{5}: \%$ data for gyrometer. $Y_{6}: \%$ data for magnetometer.

\begin{tabular}{|l|l|l||r|r|r|}
\hline$u_{5}$ & $u_{6}$ & $u_{7}$ & $Y_{4}$ & $Y_{5}$ & $Y_{6}$ \\
\hline \hline ON & L5 & None & 188.4077 & 26.427058 & 195.5040 \\
\hline ON & L5 & Thin & 195.5083 & 25.893829 & 195.4962 \\
\hline OFF & L5 & None & 333.3137 & 43.178598 & 333.4007 \\
\hline ON & L5 & Thick & 196.6234 & 7.207227 & 196.5224 \\
\hline ON & G10 & Thick & 0.0000 & 0.000000 & 0.0000 \\
\hline ON & G10 & Thick & 0.0000 & 0.000000 & 0.0000 \\
\hline OFF & L5 & Thin & 378.3933 & 51.097817 & 378.3866 \\
\hline OFF & L5 & Thick & 378.3481 & 51.059389 & 378.1941 \\
\hline OFF & G10 & Thick & 0.0000 & 0.000000 & 0.0000 \\
\hline
\end{tabular}

can see that, as for the accelerometer, the most impacting factor is $u_{6}$, which implies that the farther the smartphone is from the sensor, the lesser the communication is. We can also see that when the factor $u_{5}$ is inactive, the response is better. As previously, $u_{7}$ has no significant impact on the integrity of gyrometric data.

\subsection{Integrity of Magnetometer Data}

The estimated effects of each factor are presented in Figure 5(c). Only the first two effects were significant (t-test: $\mathrm{p}=0.0075$ and $\mathrm{p}=0.043$ respectively). Conclusions are the same than the ones already drawn in the two previous sections.

\section{Conclusion}

This study confirms the relevance of the Quality by Design approach to identify critical factors of development and to analyze their effect on the quality responses of a medical device. In this application, QbD allowed us to find suitable operating modalities such as: sampling frequency, uploading mode and period as well as the possible number of sensors to activate. It also emphasized the possibility to work in an environment composed of several types of wall within a 5-m radius from the mobile phone. This test also stressed the ability of the proposed solution to recover data after a long $(>1 \mathrm{~h})$ period of connection loss.

Further studies on the performances of this application with the integration of a Blockchain solution for greater data integrity are on the way, to completely analyze and fine tune the solution using Quality-by-Design. It will then help to define sturdy Blockchain solutions for e-health innovative solutions. 


\section{References}

1. European Medicines Agency, "Ich q8 (r2) pharmaceutical development," 2009.

2. T. Bastogne, "Quality-by-design of nanopharmaceuticals - a state of the art," 2017.

3. D. M. McDaniel and L. M. Doherty, "Total quality management case study in a navy headquarters organization," 1990.

4. T. Pyzdek, "The six sigma," 2003.

5. J. P. Womack, D. T. Jones, and D. Roos, "The machine that changed the world: The story of lean production-toyota's secret weapon in the global car wars that is now revolutionizing world industry," 2007.

6. G. A. Lewis, D. Mathieu, and R. Phan-Tan-Luu, Pharmaceutical Experimental Design. Marcel Dekker, 2005.

7. K. C. Wong, "Using an ishikawa diagram as a tool to assist memory and retrieval of relevant medical cases from the medical literature," 2011.

8. J. Lawson, Design and Analysis of Experiments with R. CRC Press, 2015. 


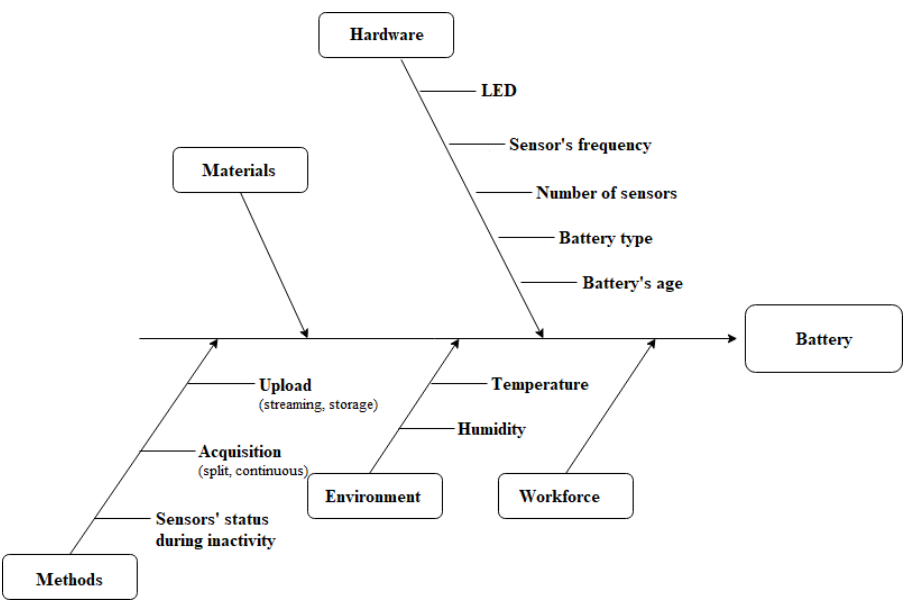

(a) List of Factors affecting the Battery Duration

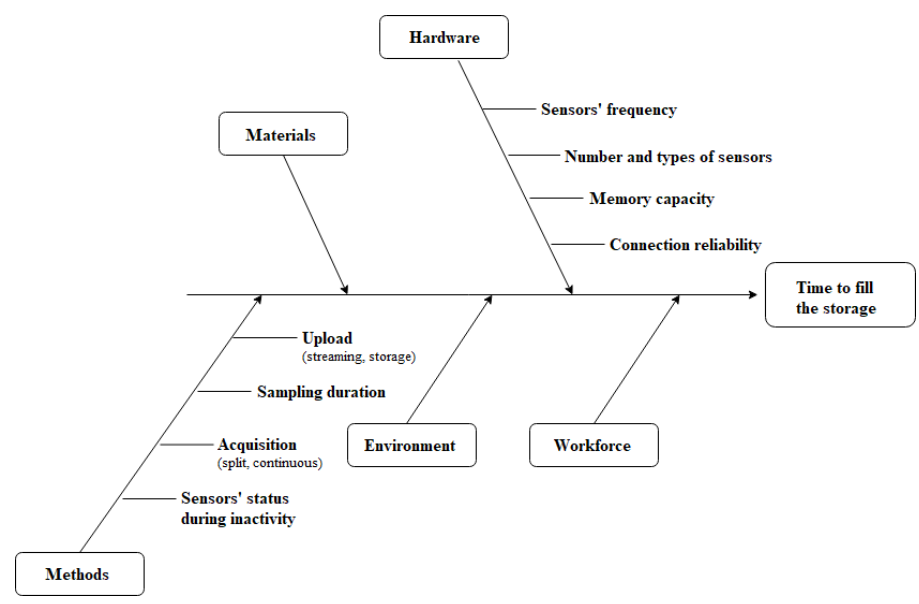

(b) List of Factors affecting the Memory Storage

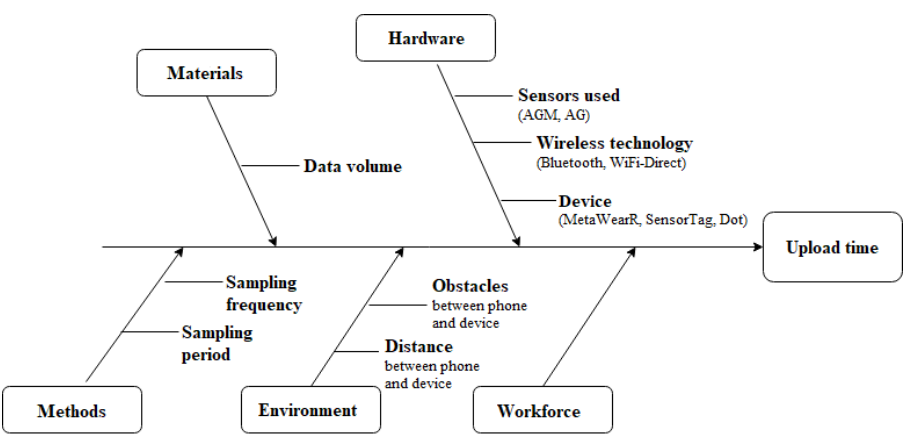

(c) List of Factors affecting the Data Uploading Duration

Fig. 3. Ishikawa diagrams of three critical quality attributes. 


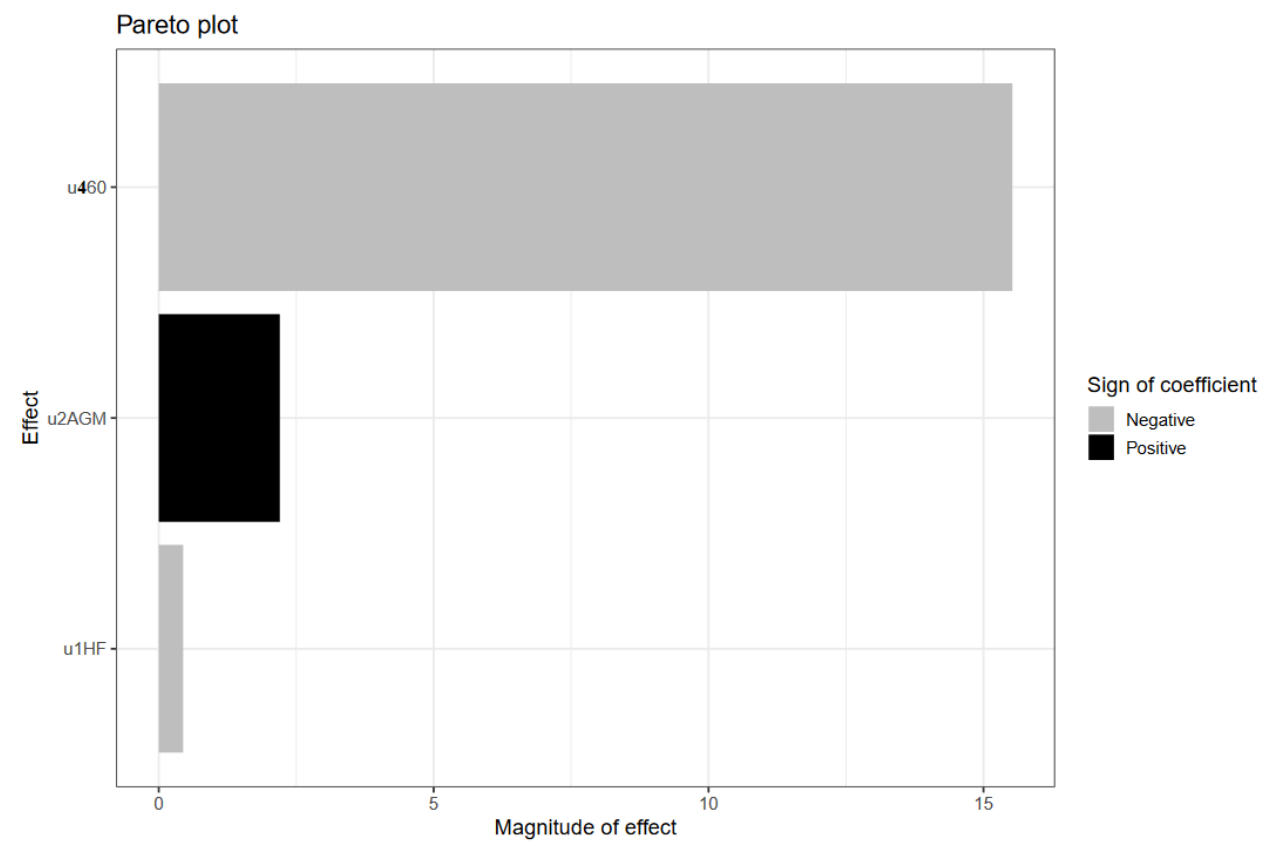

Fig. 4. Pareto Diagram of the effects on the Data Uploading Duration. P-values: 8.51e$05 ; 0.427 ; 0.871$. 


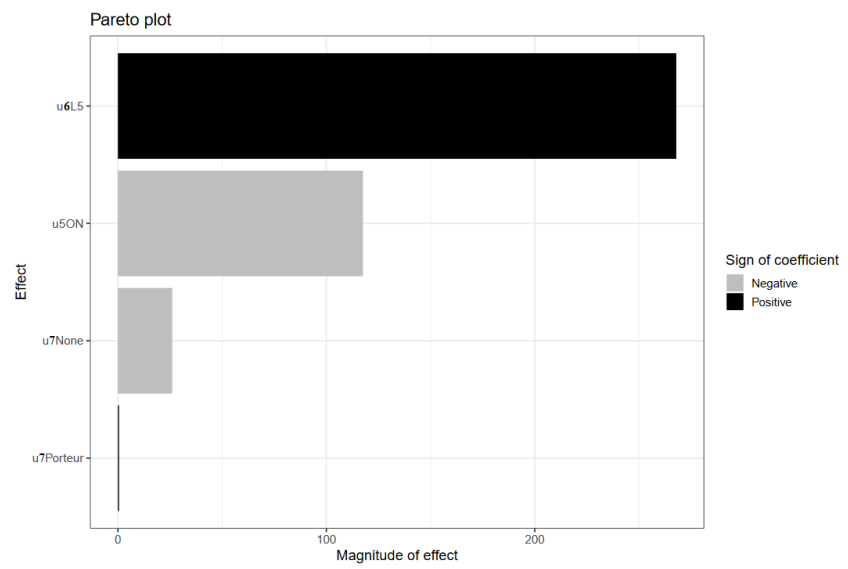

(a) Effects on the accelerometry response

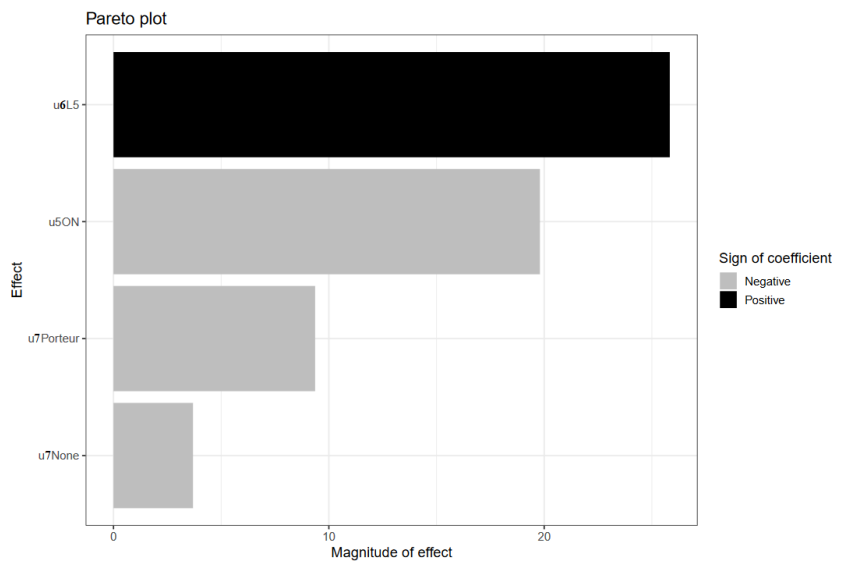

(b) Effects on the gyrometry response

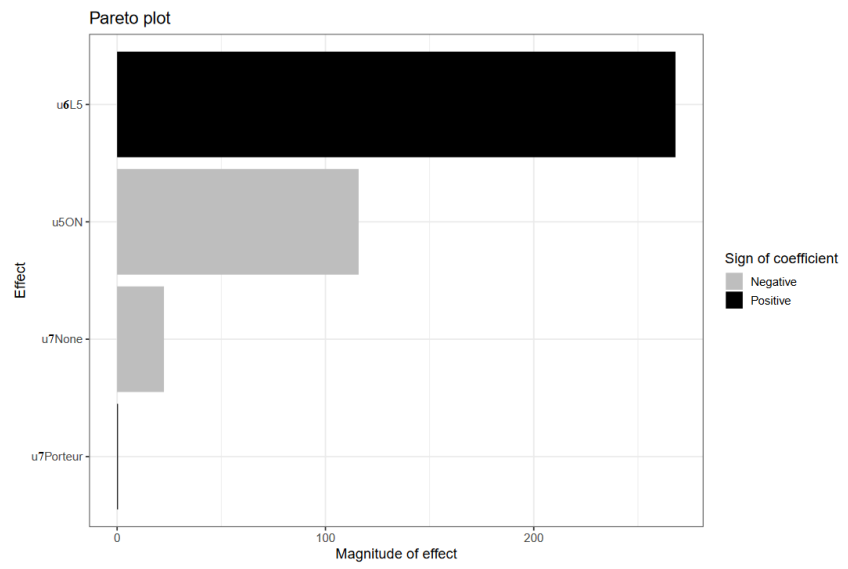

(c) Effects on the magnetometry response

Fig. 5. Pareto diagrams of the effects of three factors $\left(u_{5}\right.$ : bluetooth mode; $u_{6}$ sensor/mobile phone distance; $u_{7}$ : wall type) on the three motion responses. 